\title{
Frames of $p$-adic wavelets and orbits of the affine group
}

\author{
S.Albeverio, S.V.Kozyrev
}

October 24, 2018

\begin{abstract}
The general construction of frames of $p$-adic wavelets is described. We consider the orbit of a generic mean zero locally constant function with compact support (mean zero test function) with respect to the action of the $p$-adic affine group and show that this orbit is a uniform tight frame. We discuss the relations of this result with the multiresolution wavelet analysis.
\end{abstract}

Keywords: $p$-adic wavelets, frames of wavelets, multiresolution analysis

AMS 2000 Mathematics Subject Classification: 42C40 (Wavelets)

\section{Introduction}

Wavelet analysis (see e.g. [1], 2], [3, 4]) is the important method used in a wide variety of applications from the theory of functions to signal analysis.

A basis of wavelets in spaces of complex valued functions of a $p$-adic argument was introduced and its relation to the spectral analysis of $p$-adic pseudodifferential operators was investigated in [5]. Other examples of $p$-adic wavelet bases were considered in [6], [7], 8], [9], [10]. The relation between the continuous and the discrete $p$-adic wavelet transform was discussed in [11].

In the present paper we introduce the following general construction of frames of $p$-adic wavelets. We act on an arbitrary function $f$ in $D_{0}\left(\mathbb{Q}_{p}\right)$ (mean zero locally constant functions with compact support in the field $\mathbb{Q}_{p}$ of $p$-adic numbers) by representations from the affine group (translations and dilations):

$$
G(a, b) f(x)=f^{a, b}(x)=|a|_{p}^{-\frac{1}{2}} f\left(\frac{x-b}{a}\right),
$$

where $a, b \in \mathbb{Q}_{p}$ and $a \neq 0,|a|_{p}$ being the $p$-adic norm.

Due to local constancy of $f$ the orbit of $f$ will be a discrete set of functions. We will show that, when the function $f$ is generic (see the definition below), the orbit of $f \in D_{0}\left(\mathbb{Q}_{p}\right)$ is a tight uniform frame, i.e. $\exists A>0: \forall g \in L^{2}\left(\mathbb{Q}_{p}\right)$ :

$$
\sum_{N}\left|\left\langle f^{(N)}, g\right\rangle\right|^{2}=A\|g\|^{2},
$$


where $f^{(N)}$ are elements of the orbit of $f,\left\|f^{(N)}\right\|=\|f\|,\|\cdot\|$ is the $L^{2}$-norm.

In short the construction is as follows. Any function $f \in D_{0}\left(\mathbb{Q}_{p}\right)$ has the form of a finite linear combination of $p$-adic complex valued wavelets $\psi_{\gamma n j}$ :

$$
f=\sum_{\gamma n j} C_{\gamma n j} \psi_{\gamma n j}, \quad C_{\gamma n j} \in \mathbb{C}, \quad \psi_{\gamma n j}: \mathbb{Q}_{p} \rightarrow \mathbb{C}
$$

Then, we make several observations:

1) An orbit (with respect to the action of the affine group) of a wavelet $\psi_{\gamma n j}$ is a set $\left\{e^{2 \pi i p^{-1} m} \psi_{\gamma^{\prime} n^{\prime} j^{\prime}}\right\}$ of all products of wavelets and roots of one of degree $p$;

2) The stabilizer of a wavelet $\psi_{\gamma n j}$ can be easily computed;

3) We say that the function $f \in D_{0}\left(\mathbb{Q}_{p}\right)$ is generic, if the stabilizer of action of the affine group on $f$ is an intersection of stabilizers of wavelets which give contribution to the above expansion of $f$. In this case the stabilizer of $f$ can be easily computed;

4) Using the computed stabilizer of $f$, it is possible to construct the set of representatives of the orbit $G f$ (i.e. to parameterize the orbit);

5 ) Using the constructed parametrization of the orbit $G f$, one can check that the orbit is a tight frame.

At the step 3 of our construction it is crucially important that due to ultrametricity we can easily compute intersections, say intersection of any finite number of $p$-adic balls is a ball (in the real case an intersection of balls might have a quite complicated structure).

We discuss the relation of the introduced construction of $p$-adic frames and multiresolution analysis. We show that the multiresolution construction in $p$-adic analysis corresponds to the natural parametrization of the orbit of action of the affine group on some mean zero test function, and that the multiresolution construction possesses a family of generalizations (which correspond to orbits of arbitrary mean zero test functions).

The results of the present paper support the point of view [11] that the main constructions of wavelet analysis arise automatically as the properties of orbits of the $p$-adic affine group and, moreover, the real wavelet analysis should be considered as an analogue of these natural constructions of $p$-adic analysis.

The structure of the present paper is as follows.

In Section 2 we describe the stabilizers and orbits with respect to the action of the affine group of generic functions in $D_{0}\left(\mathbb{Q}_{p}\right)$.

In Section 3 we prove that the orbit of a generic function $f \in D_{0}\left(\mathbb{Q}_{p}\right)$ is a tight uniform frame.

In Section 4 we compare the structure of the constructed frame and the definition of multiresolution analysis and discuss the relation of muliresolution analysis with projective limits of $p$-adic groups.

In Section 5 (the Appendix) we describe the necessary definitions and notations.

\section{Orbits of the affine group}

If the function $f$ is a test function in $D\left(\mathbb{Q}_{p}\right)$, then the orbit $G f$ of the function $f$ with respect to the action of the affine group is a discrete set of test functions. In particular, the following results take place. 


\section{Lemma 1}

1) The orbit $G \Omega\left(|\cdot|_{p}\right)$ is the set of all normed characteristic functions of balls in $\mathbb{Q}_{p}$ and is in one to one correspondence with the set of functions

$$
G\left(p^{-\gamma}, p^{-\gamma} n\right) \Omega\left(|x|_{p}\right)=p^{-\frac{\gamma}{2}} \Omega\left(\left|p^{\gamma} x-n\right|_{p}\right), \quad n \in \mathbb{Q}_{p} / \mathbb{Z}_{p} .
$$

2) The stabilizer $G_{p^{-\frac{\gamma}{2}} \Omega\left(p^{\gamma \cdot-n)}\right.}$ of $p^{-\frac{\gamma}{2}} \Omega\left(p^{\gamma} \cdot-n\right)$ contains all $g=(a, b)$ with $|a|_{p}=1$ and $b$ of the form

$$
b=p^{-\gamma}(n(1-a)+z), \quad z \in \mathbb{Z}_{p} .
$$

In particular, the stabilizer of the unit ball $G_{\Omega}$ consists of $b \in \mathbb{Z}_{p}$ and $a$ with $|a|_{p}=1$.

Here we assume that the elements of the factor group $\mathbb{Q}_{p} / \mathbb{Z}_{p}$ are given by the representatives (24) in the corresponding equivalence classes.

Proof It is obvious that the affine group maps a ball into a ball and is transitive on the balls. The first statement of the lemma follows from the observation that the radii of the balls in $\mathbb{Q}_{p}$ take values $p^{\gamma}, \gamma \in \mathbb{Z}$, and the centers of the balls of the radius $p^{\gamma}$ can be chosen in the points $p^{-\gamma} n, n \in \mathbb{Q}_{p} / \mathbb{Z}_{p}$.

Let us compute the stabilizer of $\Omega\left(\left|p^{\gamma} x-n\right|_{p}\right)$. For $g=(a, b) \in G_{\Omega\left(\left|p^{\gamma}-n\right|_{p}\right)}$ we should have $|a|_{p}=1$, because for $g$ in the stabilizer the action should not change the diameter of the ball.

The point $p^{-\gamma} n$ belongs to the ball under consideration. The image of this point with respect to the transformation from the affine group is $\left(p^{-\gamma} n-b\right) / a$. Therefore the necessary (and sufficient) condition for $g=(a, b)$ to lie in the stabilizer is

$$
\left|\frac{p^{-\gamma} n-b}{a}-p^{-\gamma} n\right|_{p} \leq p^{\gamma}
$$

or

$$
\left|p^{-\gamma} n(1-a)-b\right|_{p} \leq p^{\gamma} .
$$

Therefore the stabilizer $G_{\Omega\left(p^{\gamma \cdot-n)}\right.}$ contains all $g=(a, b)$ with $|a|_{p}=1$ and $b$ of the form

$$
b=p^{-\gamma}(n(1-a)+z), \quad z \in \mathbb{Z}_{p} .
$$

In particular, the stabilizer of the unit ball $G_{\Omega}$ consists of $b \in \mathbb{Z}_{p}$ and $a$ with $|a|_{p}=1$.

This finishes the proof of the lemma.

The next lemma describes the action of the affine group on $p$-adic wavelets. The first statement of the lemma was proven in [11].

\section{Lemma 2}

1) The orbit $G \psi(\cdot)$, where $\psi$ is the $p$-adic wavelet:

$$
\psi(x)=\chi\left(p^{-1} x\right) \Omega\left(|x|_{p}\right),
$$

coincides with the set of products of functions from the wavelet basis (23) and roots of 1 of degree $p$ :

$$
G \psi=\left\{e^{2 \pi i p^{-1} m} \psi_{\gamma n j}\right\}, \quad m=0,1, \ldots, p-1, \quad \gamma \in \mathbb{Z}, \quad n \in \mathbb{Q}_{p} / \mathbb{Z}_{p}, \quad j=1, \ldots, p-1 ;
$$


where

$$
\begin{gathered}
G(a, b) \psi=e^{2 \pi i p^{-1} m} \psi_{\gamma n j}, \quad \gamma=\log _{p}|a|_{p}, \quad j=\left(a|a|_{p}\right)^{-1} \bmod p, \\
n=\left\{|a|_{p} b\right\}, \quad m=\left(\left[\left(a|a|_{p}\right)^{-1} \bmod p\right]\left(\left\{|a|_{p} b\right\}-|a|_{p} b\right)\right) \bmod p .
\end{gathered}
$$

The orbit is in one to one correspondence with the set of functions

$$
G\left(p^{-\gamma} j^{-1}, p^{-\gamma}\left(n-m j^{-1}\right)\right) \psi(x)=e^{2 \pi i p^{-1} m} \psi_{\gamma n j}(x) .
$$

2) The stabilizer $G_{\psi_{\gamma n j}}$ of the wavelet $\psi_{\gamma n j}$ contains all $g=(a, b)$ with

$$
a=1 \bmod p, \quad p^{\gamma} b=n(1-a) \bmod p .
$$

Proof 1) Let us investigate the action of the affine group on the wavelet $\psi$. We have

$$
\psi^{a, b}(x)=|a|_{p}^{-\frac{1}{2}} \psi\left(\frac{x-b}{a}\right)=|a|_{p}^{-\frac{1}{2}} \chi\left(p^{-1} \frac{x-b}{a}\right) \Omega\left(\left|\frac{x-b}{a}\right|_{p}\right) .
$$

Setting $|a|_{p}=p^{\gamma}$ we get

$$
p^{-\frac{\gamma}{2}} \chi\left(p^{-1} \frac{p^{\gamma} x-p^{\gamma} b}{a|a|_{p}}\right) \Omega\left(\left|p^{\gamma} x-p^{\gamma} b\right|_{p}\right) .
$$

Because for $a \neq 0$ we have $\left.\left.|a| a\right|_{p}\right|_{p}=1$, we can set $\left(a|a|_{p}\right)^{-1}=j+z$, where $j=1, \ldots p-1$ and $|z|_{p}<1$ (equivalently, $j=\left(a|a|_{p}\right)^{-1} \bmod p$ ). Since the character $\chi$ is locally constant and the argument of the character satisfies $\left|p^{\gamma} x-p^{\gamma} b\right| \leq 1$, we get

$$
p^{-\frac{\gamma}{2}} \chi\left(p^{-1} j\left(p^{\gamma} x-p^{\gamma} b\right)\right) \Omega\left(\left|p^{\gamma} x-p^{\gamma} b\right|_{p}\right) .
$$

Let us set $p^{\gamma} b=n-j^{-1} m+\zeta, n \in \mathbb{Q}_{p} / \mathbb{Z}_{p}, m=0,1, \ldots, p-1,|\zeta|_{p}<1$ and with $j$ as above. We get

$$
e^{2 \pi i p^{-1} m} p^{-\frac{\gamma}{2}} \chi\left(p^{-1} j\left(p^{\gamma} x-n\right)\right) \Omega\left(\left|p^{\gamma} x-n\right|_{p}\right)=e^{2 \pi i p^{-1} m} \psi_{\gamma n j}(x),
$$

where

$\gamma=\log _{p}|a|_{p}, \quad j=\left(a|a|_{p}\right)^{-1} \bmod p, \quad n=\left\{|a|_{p} b\right\}, \quad m=\left(\left[\left(a|a|_{p}\right)^{-1} \bmod p\right]\left(\left\{|a|_{p} b\right\}-|a|_{p} b\right)\right) \bmod p$.

A direct computation gives

$$
\begin{gathered}
G\left(p^{-\gamma} j^{-1}, p^{-\gamma}\left(n-m j^{-1}\right)\right) \psi(x)=p^{-\frac{\gamma}{2}} \psi\left(p^{\gamma} j x-j n+m\right)= \\
=p^{-\frac{\gamma}{2}} \chi\left(p^{-1}\left(p^{\gamma} j x-j n+m\right)\right) \Omega\left(\left|p^{\gamma} j x-j n+m\right|_{p}\right)=e^{2 \pi i p^{-1} m} \psi_{\gamma n j}(x) .
\end{gathered}
$$

2) Let us compute the stabilizer $G_{\psi_{\gamma n j}}$. We have

$$
G(a, b) \psi_{\gamma n j}(x)=p^{-\frac{\gamma}{2}} \chi\left(p^{-1} j\left(p^{\gamma} \frac{x-b}{a}-n\right)\right) \Omega\left(\left|p^{\gamma} \frac{x-b}{a}-n\right|_{p}\right) \text {. }
$$

The diameter of the support of the wavelet is invariant iff $|a|_{p}=1$. The index $j$ of the wavelet is invariant if we assume $a|a|_{p}=1$. Therefore conservation of the indices $\gamma$ and $j$ implies for $a$ the condition

$$
a=1 \bmod p .
$$


Conservation of the support of the wavelet (of index $n$ ) implies

$$
p^{\gamma} b=n(1-a) \bmod 1 \text {. }
$$

Conservation of the first multiplier in the wavelet (of the character) gives the condition

$$
p^{\gamma} b=n(1-a) \bmod p .
$$

This finishes the proof of the lemma.

Let the test function $f \in D_{0}\left(\mathbb{Q}_{p}\right)$ possess the following (finite) expansion over $p$-adic wavelets:

$$
f=\sum_{\gamma n j} C_{\gamma n j} \psi_{\gamma n j}, \quad C_{\gamma n j} \in \mathbb{C} .
$$

We say that the function $f$ is generic, if the stabilizer of action of the affine group on $f$ is an intersection of stabilizers of wavelets which give contribution to the above expansion of $f$.

Example The example of a non-generic test function can be constructed as follows. Consider the wavelet $\psi_{-1, p^{-1}, j}$ (where $j$ can take values $1, \ldots, p-1$ ) with the support in the ball $x:|x-1|_{p} \leq$ $p^{-1}$. Consider the transformation $G(a, b)$ from the affine group with $b=0, a:|a|_{p}=1,|a-1|_{p}=1$, $a^{p-1}=1 \bmod p^{2}$ (for the proof of the existence of $a$ satisfying the last condition see the discussion below). Let us define

$$
f(x)=\sum_{i=0}^{p-2} G^{i}(a, 0) \psi_{-1, p^{-1}, j}(x)=\sum_{i=0}^{p-2} \psi\left(j p^{-1}\left(\frac{x}{a^{i}}-1\right)\right)
$$

(i.e. $f$ is the sum of wavelets with supports in the maximal subballs of the sphere $|x|_{p}=1$ given by iterations of the action of $G(a, 0)$ on $\left.\psi_{-1, p^{-1}, j}\right)$.

It is easy to see that $G(a, 0)$ does not stabilize any of wavelets in (2).

Let us prove that the function $f$ is invariant with respect to the action of the described generator $G(a, 0)$. Apply $G(a, 0)$ to $f$. We get

$$
G^{i}(a, 0) f(x)=\sum_{i=1}^{p-1} \psi\left(j p^{-1}\left(\frac{x}{a^{i}}-1\right)\right) .
$$

We will have $G(a, 0) f=f$ if

$$
\psi\left(j p^{-1}\left(\frac{x}{a^{p-1}}-1\right)\right)=\psi\left(j p^{-1}(x-1)\right) .
$$

By the small Fermat theorem we have $a^{p-1}=1 \bmod p$. Moreover, by the Hensel's lemma, since the derivative $\left(a^{p-1}-1\right)^{\prime}=(p-1) a^{p-2} \neq 0 \bmod p$ for $|a|_{p}=1$, there exists $a,|a|_{p}=1$, such that $a^{p-1}=1 \bmod p^{2}$. Since the function $\psi\left(j p^{-1}(x-1)\right)$ is locally constant with the diameter of local constancy equal to $p^{-2}$, this proves that $f$ is invariant.

We see that this stabilization of the $f$ by action of the $G(a, 0)$ is related with non-genericity of the $f$ : if we multiply the wavelets in (2) by generic coefficients, the obtained function will not be stabilized by the action of $G(a, 0)$.

The next lemma will give the parametrization of the action of the affine group on an arbitrary generic test function in $D_{0}\left(\mathbb{Q}_{p}\right)$. 
Lemma 3 Assume that the function $f \in D_{0}\left(\mathbb{Q}_{p}\right)$ given by (11) is generic. Then:

1) The stabilizer $G_{f}$ of the action of the affine group on $f$ given by (11) consists of $g \in G$, $g=(a, b):$ a belongs to the ball

$$
|1-a|_{p} \leq p^{-\gamma_{A}}=\min \left[p^{-1}, \frac{\max \left(p^{\gamma_{i}-1}, p^{\gamma_{j}-1}\right)}{\left|p^{-\gamma_{i}} n_{i}-p^{-\gamma_{j}} n_{j}\right|_{p}}\right],
$$

where the minimum is taken over all $\left(\gamma_{i}, n_{i}\right)$ and $\left(\gamma_{j}, n_{j}\right)$ in (1) for which $p^{-\gamma_{i}} n_{i}-p^{-\gamma_{j}} n_{j} \neq 0$, and $b$ satisfies

$$
\left|b-p^{-\gamma_{0}} n_{0}(1-a)\right|_{p} \leq p^{\gamma_{0}-1},
$$

where $\gamma_{0}$ is the minimal $\gamma$ in the expansion (11) and $n_{0}$ is the corresponding $n$.

2) The orbit $G f$ is in one to one correspondence with the set of functions

$$
\begin{gathered}
\left\{G\left(p^{\gamma} J, p^{\gamma} J n\right) f\right\}, \quad \gamma \in \mathbb{Z}, \quad n \in \mathbb{Q}_{p} / p^{1-\gamma_{0}} \mathbb{Z}_{p}, \\
J=j+j_{1} p+j_{2} p^{2}+\cdots+j_{\gamma_{A}-1} p^{\gamma_{A}-1}, \quad j=1, \ldots, p-1, \quad j_{i}=0, \ldots, p-1 .
\end{gathered}
$$

Let us note that the indices $J$ form a group with respect to multiplication $\bmod p^{\gamma_{A}}$.

Proof 1) All the functions in the linear combination (11) are mutually orthogonal. Since the action of the affine group is unitary, it conserves the orthogonality.

Therefore the stabilizer of $f$ is the intersection of the stabilizers of all the functions in the above linear combination. By lemma 2 we get that $g=(a, b)$ lies in the stabilizer $G_{f}$ iff

$$
a=1 \bmod p, \quad p^{\gamma} b=n(1-a) \bmod p
$$

for all pairs $(\gamma, n)$ giving nonzero contributions $C_{\gamma n j}$ to (1).

Let us fix two pairs $\left(\gamma_{1}, n_{1}\right)$ and $\left(\gamma_{2}, n_{2}\right)$. We choose $\gamma_{1} \leq \gamma_{2}$. We describe the intersection of the corresponding sets defined by (7), i.e. the solution of the system

$$
a=1 \bmod p, \quad p^{\gamma_{1}} b=n_{1}(1-a) \bmod p, \quad p^{\gamma_{2}} b=n_{2}(1-a) \bmod p .
$$

For the fixed $a$ the sets of solutions of both equations for $b$ are the balls of the diameters $p^{\gamma_{1}-1}$, $p^{\gamma_{2}-1}$ and the centers $p^{-\gamma_{1}} n_{1}(1-a), p^{-\gamma_{2}} n_{2}(1-a)$ correspondingly.

Due to the strong triangle inequality these balls have nonempty intersection if

$$
\left|\left(p^{-\gamma_{1}} n_{1}-p^{-\gamma_{2}} n_{2}\right)(1-a)\right|_{p} \leq \max \left(p^{\gamma_{1}-1}, p^{\gamma_{2}-1}\right)=p^{\gamma_{2}-1} .
$$

If this condition is satisfied then $b$ belongs to the ball of the diameter $p^{\gamma_{1}-1}$ with the center in $p^{-\gamma_{1}} n_{1}(1-a)$.

We have proved that the solution of the system (8) consists of $a$ and $b$ satisfying

$$
|1-a|_{p}<1, \quad|1-a|_{p} \leq \frac{p^{\gamma_{2}-1}}{\left|p^{-\gamma_{1}} n_{1}-p^{-\gamma_{2}} n_{2}\right|_{p}}, \quad\left|b-p^{-\gamma_{1}} n_{1}(1-a)\right|_{p} \leq p^{\gamma_{1}-1},
$$

if $p^{-\gamma_{1}} n_{1}-p^{-\gamma_{2}} n_{2} \neq 0$, and

$$
|1-a|_{p}<1, \quad\left|b-p^{-\gamma_{1}} n_{1}(1-a)\right|_{p} \leq p^{\gamma_{1}-1},
$$


if $p^{-\gamma_{1}} n_{1}-p^{-\gamma_{2}} n_{2}=0$.

In general, consider the system of equations (7) for all pairs $(\gamma, n)$ giving nonzero contributions to (11). The solution of this system consists of $a$, satisfying

$$
|1-a|_{p} \leq \min \left[p^{-1}, \frac{\max \left(p^{\gamma_{i}-1}, p^{\gamma_{j}-1}\right)}{\left|p^{-\gamma_{i}} n_{i}-p^{-\gamma_{j}} n_{j}\right|_{p}}\right]
$$

where the minimum is taken over all $\left(\gamma_{i}, n_{i}\right)$ and $\left(\gamma_{j}, n_{j}\right)$ for which $p^{-\gamma_{i}} n_{i}-p^{-\gamma_{j}} n_{j} \neq 0$, and of $b$, satisfying

$$
\left|b-p^{-\gamma_{0}} n_{0}(1-a)\right|_{p} \leq p^{\gamma_{0}-1},
$$

where $\gamma_{0}$ is the minimal $\gamma$ in the expansion (11) and $n_{0}$ is the corresponding $n$ (which, in general, is not uniquely defined, but the corresponding set of $b$ is defined unambiguously).

2) Let us construct the parametrization of the orbit $G f$ of the function $f \in D_{0}\left(\mathbb{Q}_{p}\right)$ with respect to the action of the affine group.

Consider the subgroup of dilations in the affine group (transformations with $b=0$ ) and the system of representatives in the orbit of $f$ with respect to transformations from this subgroup. This system by (3) will be given by

$\left\{G\left(p^{\gamma} J, 0\right)\right\}, \quad \gamma \in Z, \quad J=j+j_{1} p+j_{2} p^{2}+\cdots+j_{\gamma_{A}-1} p^{\gamma_{A}-1}, \quad j=1, \ldots, p-1, \quad j_{i}=0, \ldots, p-1$.

Here $\gamma_{A}$ is given by (3).

Let us introduce the parametrization of the orbit of $f$ by

$$
\left\{G\left(p^{\gamma} J, p^{\gamma} J n\right) f\right\}, \quad \gamma \in \mathbb{Z}, \quad n \in \mathbb{Q}_{p} / p^{1-\gamma_{0}} \mathbb{Z}_{p}
$$

and where $J$ is as in (9).

To prove that the above formula indeed gives the parametrization of the orbit $G f$ of the affine group it is sufficient to show that any transformation from the affine group possesses the unique expansion

$$
G(a, b)=G\left(a_{1}, b_{1}\right) G\left(a_{0}, b_{0}\right)=G\left(p^{\gamma} J, p^{\gamma} J n\right) G\left(a_{0}, b_{0}\right),
$$

where $G\left(a_{0}, b_{0}\right)$ lies in the stabilizer of $f$ and $G\left(a_{1}, b_{1}\right)=G\left(p^{\gamma} J, p^{\gamma} J n\right)$ is the representative of the orbit in formula (10) (therefore $\left.G(a, b) f=G\left(a_{1}, b_{1}\right) f\right)$.

To show this we define $\gamma, J, n$ in (10) as

$$
\gamma=\log _{p}|a|_{p}^{-1}, \quad J=a|a|_{p} \bmod p^{\gamma_{A}}, \quad n=\left(\left(b a_{1}^{-1}\right) \bmod p^{1-\gamma_{0}}\right),
$$

where $a_{1}=p^{\gamma} J$.

We have to prove that:

i) $G\left(a_{0}, b_{0}\right)=G^{-1}\left(a_{1}, b_{1}\right) G(a, b)$ lies in the stabilizer of $f$;

ii) for the different representatives of the orbit $G\left(a_{1}, b_{1}\right), G\left(a_{1}^{\prime}, b_{1}^{\prime}\right)$ the combination

$$
G^{-1}\left(a_{1}, b_{1}\right) G\left(a_{1}^{\prime}, b_{1}^{\prime}\right)
$$

can not belong to the stabilizer.

i) Since

$$
G\left(a_{0}, b_{0}\right)=G^{-1}\left(a_{1}, b_{1}\right) G(a, b)=G\left(a_{1}^{-1} a,\left(b-b_{1}\right) a_{1}^{-1}\right),
$$


we have

$$
\begin{gathered}
a_{0}=a_{1}^{-1} a=a|a|_{p}\left(a|a|_{p} \bmod p^{\gamma_{A}}\right)^{-1}=1 \bmod p^{\gamma_{A}} ; \\
b_{0}=\left(b-b_{1}\right) a_{1}^{-1}=b a_{1}^{-1}-\left(\left(b a_{1}^{-1}\right) \bmod p^{1-\gamma_{0}}\right)=0 \bmod p^{1-\gamma_{0}} .
\end{gathered}
$$

Therefore $G\left(a_{0}, b_{0}\right)$ lies in the stabilizer.

ii) Since

$$
G^{-1}\left(a_{1}, b_{1}\right) G\left(a_{1}^{\prime}, b_{1}^{\prime}\right)=G\left(a_{1}^{-1} a_{1}^{\prime},\left(b_{1}^{\prime}-b_{1}\right) a_{1}^{-1}\right),
$$

the above combination belongs to the stabilizer if

$$
\begin{gathered}
a_{1}^{-1} a_{1}^{\prime}=\left(p^{\gamma} J\right)^{-1} p^{\gamma^{\prime}} J^{\prime}=1 \bmod p^{\gamma_{A}} ; \\
\left(b_{1}^{\prime}-b_{1}\right) a_{1}^{-1}=n^{\prime} a_{1}^{\prime} a_{1}^{-1}-n=0 \bmod p^{1-\gamma_{0}} ;
\end{gathered}
$$

which is possible only if

$$
\gamma=\gamma^{\prime}, \quad J=J^{\prime}, \quad n=n^{\prime} .
$$

This finishes the proof of the lemma.

Remark The obtained in the above lemma parametrization of the orbit $G f$ is not unique. For instance, one can, for each fixed $J$ and $\gamma$, choose the different representatives $n$ in the equivalence classes in $\mathbb{Q}_{p} / p^{1-\gamma_{0}} \mathbb{Z}_{p}$.

In particular, one can choose the parametrization of the elements of the orbit $G f$ in the form

$$
\left\{G\left(p^{\gamma} J, p^{\gamma} n\right) f\right\}
$$

where the indices are as in (10).

Remark For the above lemma it is crucially important that in ultrametric spaces it is easy to compute intersections. In particular, intersection of any finite number of $p$-adic balls is a ball, while in the real case an intersection of balls may have a quite complicated structure.

\section{$3 \quad$ Frames and orbits}

Consider the function $f \in D_{0}\left(\mathbb{Q}_{p}\right)$ defined by (1) and the set of representatives of action of the affine group on this function defined by (5):

$$
f^{(\gamma n J)}=G\left(p^{\gamma} J, p^{\gamma} J n\right) f .
$$

We have the following lemma1:

Lemma 4 For $f \in D_{0}\left(\mathbb{Q}_{p}\right)$ given by (11) the following series is proportional to the identity operator and is equal to

$$
S=\sum_{\gamma n J}\left|f^{(\gamma n J)}\right\rangle\left\langle\left. f^{(\gamma n J)}\left|=\sum_{\gamma_{1} n_{1} j_{1}}\right| C_{\gamma_{1} n_{1} j_{1}}\right|^{2} p^{\gamma_{A}-\gamma_{0}+\gamma_{1}}\right.
$$

\footnotetext{
${ }^{1}$ Here we use the standard notations from quantum mechanics: $|\psi\rangle$ denotes the element of the Hilbert space $L^{2}\left(\mathbb{Q}_{p}\right)$ given by the function $\psi,\langle\psi|$ is the canonically conjugate linear bounded functional on $L^{2}\left(\mathbb{Q}_{p}\right)$, and $|\psi\rangle\langle\phi|$, $\|\psi\|=\|\phi\|=1$, is the operator in $L^{2}\left(\mathbb{Q}_{p}\right)$ which maps $\phi$ onto $\psi$ and kills the orthogonal complement to $\phi$.
} 
(i.e. $S$ is an operator of multiplication by a number). The summation over $\gamma, n, J$ runs over the parameters described by (5), (6). The summation over $\gamma_{1}, n_{1}, j_{1}$ runs over the indices of the wavelet basis. The $\gamma_{A}$ is given by (3), $\gamma_{0}$ is the minimal $\gamma$ in (11).

The convergence of the series over $\gamma, n, J$ is understood as the convergence of matrix elements in the wavelet basis (i.e. as the weak convergence).

Proof Since

$$
f=\sum_{\gamma n j} C_{\gamma n j} \psi_{\gamma n j}
$$

we have

$$
\begin{gathered}
S=\sum_{\gamma n J} G\left(p^{\gamma} J, p^{\gamma} J n\right)|f\rangle\langle f| G^{*}\left(p^{\gamma} J, p^{\gamma} J n\right)= \\
=\sum_{\gamma_{1} n_{1} j_{1} ; \gamma_{2} n_{2} j_{2}} C_{\gamma_{1} n_{1} j_{1}} C_{\gamma_{2} n_{2} j_{2}}^{*} \sum_{\gamma n J} G\left(p^{\gamma} J, p^{\gamma} J n\right)\left|\psi_{\gamma_{1} n_{1} j_{1}}\right\rangle\left\langle\psi_{\gamma_{2} n_{2} j_{2}}\right| G^{*}\left(p^{\gamma} J, p^{\gamma} J n\right)
\end{gathered}
$$

We can change the order of the summation because the summation over $\left(\gamma_{1} n_{1} j_{1}\right),\left(\gamma_{2} n_{2} j_{2}\right)$ is finite and the series over $(\gamma n J)$ applied to any wavelet gives a finite number of non-vanishing terms.

Let us check that in the above sum over $\left(\gamma_{1} n_{1} j_{1}\right),\left(\gamma_{2} n_{2} j_{2}\right)$ only diagonal terms (i.e. terms with $\gamma_{1}=\gamma_{2}, n_{1}=n_{2}, j_{1}=j_{2}$ ) give non-zero contributions.

Consider the sum over $n$ (all the other indices are fixed):

$$
\begin{gathered}
\sum_{n \in \mathbb{Q}_{p} / p^{1-\gamma_{0} \mathbb{Z}_{p}}} G\left(p^{\gamma} J, p^{\gamma} J n\right)\left|\psi_{\gamma_{1} n_{1} j_{1}}\right\rangle\left\langle\psi_{\gamma_{2} n_{2} j_{2}}\right| G^{*}\left(p^{\gamma} J, p^{\gamma} J n\right)= \\
=G\left(p^{\gamma} J, 0\right)\left[\sum_{n \in \mathbb{Q}_{p} / p^{1-\gamma_{0} \mathbb{Z}_{p}}} G(1, n)\left|\psi_{\gamma_{1} n_{1} j_{1}}\right\rangle\left\langle\psi_{\gamma_{2} n_{2} j_{2}}\right| G^{*}(1, n)\right] G^{*}\left(p^{\gamma} J, 0\right) .
\end{gathered}
$$

The summation here runs over

$$
n=n_{\alpha} p^{\alpha}+n_{\alpha+1} p^{\alpha+1}+\cdots+n_{-\gamma_{0}} p^{-\gamma_{0}}, \quad n_{j}=0, \ldots, p-1, \quad \alpha \leq-\gamma_{0} .
$$

Note that by construction

$$
-\gamma_{1} \leq-\gamma_{0}, \quad-\gamma_{2} \leq-\gamma_{0}
$$

we also assume $-\gamma_{1} \leq-\gamma_{2}$.

Therefore the summation over $n$ takes the form

$$
\begin{gathered}
\sum_{n \in \mathbb{Q}_{p} / p^{1-\gamma_{0}} \mathbb{Z}_{p}} G(1, n)\left|\psi_{\gamma_{1} n_{1} j_{1}}\right\rangle\left\langle\psi_{\gamma_{2} n_{2} j_{2}}\right| G^{*}(1, n)= \\
=\sum_{n \in \mathbb{Q}_{p} / p^{-\gamma_{2}} \mathbb{Z}_{p}} \sum_{n_{-\gamma_{2}}, n_{-\gamma_{2}}+1, \ldots, n_{-\gamma_{0}}} G(1, n)\left|\psi_{\gamma_{1} n_{1} j_{1}}\right\rangle\left\langle\psi_{\gamma_{2} n_{2} j_{2}}\right| G^{*}(1, n)= \\
=p^{\gamma_{0}-\gamma_{2}} \sum_{n \in \mathbb{Q}_{p} / p^{-\gamma_{2}}} \sum_{\mathbb{Z}_{p} n_{-\gamma_{2}}} G(1, n)\left|\psi_{\gamma_{1} n_{1} j_{1}}\right\rangle\left\langle\psi_{\gamma_{2} n_{2} j_{2}}\right| G^{*}(1, n) .
\end{gathered}
$$


If $-\gamma_{1}<-\gamma_{2}$, then

$$
\sum_{n_{-\gamma_{2}}} G(1, n)\left|\psi_{\gamma_{1} n_{1} j_{1}}\right\rangle\left\langle\psi_{\gamma_{2} n_{2} j_{2}}\left|G^{*}(1, n)=\right| \psi_{\gamma_{1} n_{1} j_{1}}\right\rangle\left\langle\psi_{\gamma_{2} n_{2} j_{2}}\right| \sum_{n_{-\gamma_{2}}=0, \ldots, p-1} e^{2 \pi i p^{-1} j_{2} n_{-\gamma_{2}}}=0
$$

since the sum of roots of one of the degree $p$ is equal to zero.

If $-\gamma_{1}=-\gamma_{2}$ and $j_{1} \neq j_{2}$, we get

$$
\sum_{n_{-\gamma_{1}}} G(1, n)\left|\psi_{\gamma_{1} n_{1} j_{1}}\right\rangle\left\langle\psi_{\gamma_{1} n_{2} j_{2}}\left|G^{*}(1, n)=\right| \psi_{\gamma_{1} n_{1} j_{1}}\right\rangle\left\langle\psi_{\gamma_{1} n_{2} j_{2}}\right| \sum_{n_{-\gamma_{1}}=0, \ldots, p-1} e^{2 \pi i p^{-1}\left(j_{2}-j_{1}\right) n_{-\gamma_{1}}}=0 .
$$

Therefore the only nonzero contributions in (14) correspond to contributions with $\gamma_{1}=\gamma_{2}$ and $j_{1}=j_{2}$.

Let us fix $\gamma_{1}=\gamma_{2}, j_{1}=j_{2}$ and $n_{1} \neq n_{2}$ and consider the sum

$$
\sum_{J} G(J, 0)\left|\psi_{\gamma_{1} n_{1} j_{1}}\right\rangle\left\langle\psi_{\gamma_{1} n_{2} j_{1}}\right| G^{*}(J, 0)
$$

Note that at least one of $n_{1}, n_{2}$ is non zero.

We will investigate the sum over the subgroup of $J$ which leaves invariant the supports of the both wavelets $\psi_{\gamma_{1} n_{1} j_{1}}, \psi_{\gamma_{1} n_{2} j_{1}}$ in the above expression. By the previous lemma this sum contains $J$ which satisfy for non zero $n_{1}, n_{2}$

$$
|J-1|_{p} \leq \min \left(\left|n_{1}\right|_{p}^{-1},\left|n_{2}\right|_{p}^{-1}\right)=p^{-\gamma_{B}},
$$

and, for the case when one of $n_{1}, n_{2}$ is equal to zero, say $n_{1}=0$,

$$
|J-1|_{p} \leq\left|n_{2}\right|_{p}^{-1}=p^{-\gamma_{B}} .
$$

We get for such $J$

$$
\begin{aligned}
& G(J, 0) \psi_{\gamma n j}(x)=p^{-\frac{\gamma}{2}} \chi\left(p^{-1}(j / J)\left(p^{\gamma} x-n J\right)\right) \Omega\left(\left|p^{\gamma} x-n J\right|_{p}\right)= \\
& =p^{-\frac{\gamma}{2}} \chi\left(p^{-1} j\left(p^{\gamma} x-n J\right)\right) \Omega\left(\left|p^{\gamma} x-n J\right|_{p}\right)=\psi_{\gamma n j}(x) e^{2 \pi p^{-1} j n(1-J)} .
\end{aligned}
$$

Let us compute the sum

$$
\sum_{J} G(J, 0)\left|\psi_{\gamma_{1} n_{1} j_{1}}\right\rangle\left\langle\psi_{\gamma_{1} n_{2} j_{1}}\left|G^{*}(J, 0)=\sum_{j, j_{1}, \ldots, j_{\gamma_{B}}} \sum_{j_{\gamma_{B}}} \sum_{j_{\gamma_{B}+1}, \ldots, j_{\gamma_{A}}-1} G(J, 0)\right| \psi_{\gamma_{1} n_{1} j_{1}}\right\rangle\left\langle\psi_{\gamma_{1} n_{2} j_{1}}\right| G^{*}(J, 0) .
$$

We note that by (3) we have $\gamma_{B} \leq \gamma_{A}-1$. The sum over the indices $j_{\gamma_{B}}, j_{\gamma_{B}+1}, \ldots, j_{\gamma_{A}-1}$ takes the form

$$
p^{\gamma_{A}-\gamma_{B}-1}\left|\psi_{\gamma_{1} n_{1} j_{1}}\right\rangle\left\langle\psi_{\gamma_{1} n_{2} j_{1}}\right| \sum_{j_{\gamma_{B}}=0, \ldots, p-1} e^{-2 \pi p^{-1} j_{1}\left(n_{1}-n_{2}\right) j_{\gamma_{B}}}=0 .
$$

Therefore nonzero contributions can only arise from the terms with $n_{1}=n_{2}$.

We have proved that (14) takes the form

$$
S=\sum_{\gamma_{1} n_{1} j_{1}}\left|C_{\gamma_{1} n_{1} j_{1}}\right|^{2} \sum_{\gamma n J} G\left(p^{\gamma} J, p^{\gamma} J n\right)\left|\psi_{\gamma_{1} n_{1} j_{1}}\right\rangle\left\langle\psi_{\gamma_{1} n_{1} j_{1}}\right| G^{*}\left(p^{\gamma} J, p^{\gamma} J n\right) .
$$


Let us compute for the wavelet $\psi_{\gamma_{1} n_{1} j_{1}}$ the number of transformations

$$
G\left(p^{\gamma} J, p^{\gamma} J n\right) \psi_{\gamma_{1} n_{1} j_{1}}
$$

which act as a multiplication of $\psi_{\gamma_{1} n_{1} j_{1}}$ by a root of one (and therefore leave the rank one projection operator $\left|\psi_{\gamma_{1} n_{1} j_{1}}\right\rangle\left\langle\psi_{\gamma_{1} n_{1} j_{1}}\right|$ unchanged). To obtain a multiplication of the wavelet by a constant we should take $\gamma=0$ and $j=1$ in the formula ([6) for $J$ (i.e. $J=1 \bmod p$ ). We get the transformation

$$
\begin{gathered}
G(J, J n) \psi_{\gamma_{1} n_{1} j_{1}}(x)=p^{-\frac{\gamma_{1}}{2}} \chi\left(p^{-1} \frac{j_{1}}{J}\left(p^{\gamma_{1}} x-J\left(p^{\gamma_{1}} n+n_{1}\right)\right)\right) \Omega\left(\left|p^{\gamma_{1}} x-J\left(p^{\gamma_{1}} n+n_{1}\right)\right|_{p}\right)= \\
=p^{-\frac{\gamma_{1}}{2}} \chi\left(p^{-1} j_{1}\left(p^{\gamma_{1}} x-J\left(p^{\gamma_{1}} n+n_{1}\right)\right)\right) \Omega\left(\left|p^{\gamma_{1}} x-J\left(p^{\gamma_{1}} n+n_{1}\right)\right|_{p}\right) .
\end{gathered}
$$

In order to get the support of the wavelet unchanged we need

$$
J p^{\gamma_{1}} n=(1-J) n_{1} \bmod 1
$$

or equivalently

$$
\left|J p^{\gamma_{1}} n-(1-J) n_{1}\right|_{p} \leq 1
$$

The summation runs over

$$
\begin{gathered}
n=n_{\alpha} p^{\alpha}+n_{\alpha+1} p^{\alpha+1}+\cdots+n_{-\gamma_{0}} p^{-\gamma_{0}}, \quad n_{j}=0, \ldots, p-1, \quad \alpha \leq-\gamma_{0} ; \\
J=1+j_{1} p+j_{2} p^{2}+\cdots+j_{\gamma_{A}-1} p^{\gamma_{A}-1}, \quad j_{i}=0, \ldots, p-1 .
\end{gathered}
$$

Let us compute the number of $n$ satisfying (15) for fixed $J$. Since there always exists the solution of (15) given by $n_{0}=\left(J^{-1} p^{-\gamma_{1}}(1-J) n_{1}\right) \bmod p^{1-\gamma_{0}}$, the set of solutions will be given by $n:\left|n-n_{0}\right|_{p} \leq p^{\gamma_{1}}$. There are $p^{-\gamma_{0}+\gamma_{1}+1}$ such $n$.

Since there are $p^{\gamma_{A}-1}$ possible meanings of $J$, in total we have $p^{\gamma_{A}-\gamma_{0}+\gamma_{1}}$ solutions of (15).

Since the set of transformations (5) is transitive on vectors from the basis of wavelets, the following series of transformations of the projection operator by $G\left(p^{\gamma} J, p^{\gamma} J n\right)$ is proportional to the identity operator:

$$
\sum_{\gamma n J} G\left(p^{\gamma} J, p^{\gamma} J n\right)\left|\psi_{\gamma_{1} n_{1} j_{1}}\right\rangle\left\langle\psi_{\gamma_{1} n_{1} j_{1}}\right| G^{*}\left(p^{\gamma} J, p^{\gamma} J n\right)=p^{\gamma_{A}-\gamma_{0}+\gamma_{1}}
$$

This implies for $S$ the expression

$$
S=\sum_{\gamma_{1} n_{1} j_{1}}\left|C_{\gamma_{1} n_{1} j_{1}}\right|^{2} \sum_{\gamma n J} G\left(p^{\gamma} J, p^{\gamma} J n\right)\left|\psi_{\gamma_{1} n_{1} j_{1}}\right\rangle\left\langle\left.\psi_{\gamma_{1} n_{1} j_{1}}\left|G^{*}\left(p^{\gamma} J, p^{\gamma} J n\right)=\sum_{\gamma_{1} n_{1} j_{1}}\right| C_{\gamma_{1} n_{1} j_{1}}\right|^{2} p^{\gamma_{A}-\gamma_{0}+\gamma_{1}} .\right.
$$

This finishes the proof of the lemma.

The next theorem describes frames of $p$-adic wavelets given by orbits of generic functions from $D_{0}\left(\mathbb{Q}_{p}\right)$ with respect to the action of the affine group. 
Theorem 5 Let $f \in D_{0}\left(\mathbb{Q}_{p}\right)$ is generic and let $f$ possess the expansion over wavelets

$$
f=\sum_{\gamma n j} C_{\gamma n j} \psi_{\gamma n j} ; \quad \gamma \in \mathbb{Z}, \quad n \in \mathbb{Q}_{p} / \mathbb{Z}_{p}, \quad j=1, \ldots, p-1 .
$$

Then the orbit of $f$ with respect to action of the affine group possess the following properties: 1) the orbit is given by the set of functions:

$$
f^{(\gamma n J)}=G\left(p^{\gamma} J, p^{\gamma} J n\right) f, \quad \gamma \in \mathbb{Z}, \quad n \in \mathbb{Q}_{p} / p^{1-\gamma_{0}} \mathbb{Z}_{p},
$$

$\gamma_{0}$ is the minimal $\gamma$ in (16),

$$
\begin{gathered}
J=j+j_{1} p+j_{2} p^{2}+\cdots+j_{\gamma_{A}-1} p^{\gamma_{A}-1}, \quad j=1, \ldots, p-1, \quad j_{i}=0, \ldots, p-1 ; \\
p^{-\gamma_{A}}=\min \left[p^{-1}, \frac{\max \left(p^{\gamma_{i}-1}, p^{\gamma_{j}-1}\right)}{\left|p^{-\gamma_{i}} n_{i}-p^{-\gamma_{j}} n_{j}\right|_{p}}\right],
\end{gathered}
$$

where the minimum is taken over all $\left(\gamma_{i}, n_{i}\right)$ and $\left(\gamma_{j}, n_{j}\right)$ in (16) for which $p^{-\gamma_{i}} n_{i}-p^{-\gamma_{j}} n_{j} \neq 0$;

2) the orbit is a uniform and tight frame in $L^{2}\left(\mathbb{Q}_{p}\right)$ : the norms of all $f^{(\gamma n J)}$ are equal and for any $g \in L^{2}\left(\mathbb{Q}_{p}\right)$

$$
\sum_{\gamma n J}\left|\left\langle g, f^{(\gamma n J)}\right\rangle\right|^{2}=\|g\|^{2} \sum_{\gamma n j}\left|C_{\gamma n j}\right|^{2} p^{\gamma_{A}-\gamma_{0}+\gamma} .
$$

Note that on the LHS of (18) the summation runs over the elements of the orbit of $f$ and on the RHS the summation runs over the elements of the wavelet basis.

Proof The first statement of the theorem is proven in lemma 3 ,

The second statement follows from lemma 4: for $g \in D_{0}\left(\mathbb{Q}_{p}\right)$ one can see that by lemma 4

$$
\sum_{\gamma n J}\left|\left\langle g, f^{(\gamma n J)}\right\rangle\right|^{2}=\langle g, S g\rangle=S\|g\|^{2}=\|g\|^{2} \sum_{\gamma n j}\left|C_{\gamma n j}\right|^{2} p^{\gamma_{A}-\gamma_{0}+\gamma} .
$$

Since $D_{0}\left(\mathbb{Q}_{p}\right)$ is dense in $L^{2}\left(\mathbb{Q}_{p}\right)$ this finishes the proof of the theorem.

Example 1 Consider the case $f(x)=\psi(x)=\chi\left(p^{-1} x\right) \Omega\left(|x|_{p}\right)$. In this case by lemma 2 the orbit of $f$ is given by the set $\left\{e^{2 \pi i p^{-1} m} \psi_{\gamma n j}\right\}$ of products of wavelets from the basis (23) of [5] and roots of one of the degree $p$. It is easy to see that the bound of the frame will be equal to $p$ :

$$
\sum_{\gamma n J}\left|\left\langle g, f^{(\gamma n J)}\right\rangle\right|^{2}=p\|g\|^{2} .
$$

Example 2 Consider the more general example when

$$
f=\sum_{n j} C_{n j} \psi_{\gamma n j}
$$

i.e. $f$ is equal to the linear combination of wavelets with the fixed scale $\gamma$.

In this case by (18) one gets for the bound of the corresponding frame $\left\{f^{(\gamma n J)}\right\}$ the expression

$$
p^{\gamma_{A}} \sum_{n j}\left|C_{n j}\right|^{2}=p^{\gamma_{A}}\|f\|^{2}
$$

where $\gamma_{A} \geq 1$ (the minimal $\gamma_{A}=1$ was considered in the previous example when $f=\psi$ ).

Note that all examples of $p$-adic wavelet bases built in [8], 9] correspond to the case (19). 


\section{Relation to the multiresolution analysis}

The frame $\left\{G\left(p^{\gamma} J, p^{\gamma} J n\right) f\right\}$ described in theorem 5, is constructed by translations (which correspond to the index $n$ ) and dilations (described by the indices $\gamma$ and $J$ ) of the generic function $f \in D_{0}\left(\mathbb{Q}_{p}\right)$. Therefore it is natural to discuss the set $\left\{G\left(p^{\gamma} J, p^{\gamma} J n\right) f\right\}$ as a frame of wavelets.

By definition, a frame of $p$-adic wavelets is given by the set of translations and dilations

$$
G\left(p^{\gamma_{1}}, p^{\gamma_{1}} n_{1}\right) f^{\left(J_{1}\right)}, \quad \gamma_{1} \in \mathbb{Z}, \quad n_{1} \in \mathbb{Q}_{p} / \mathbb{Z}_{p},
$$

and $\left\{f^{\left(J_{1}\right)}\right\}$ is a finite set of mean zero functions in $L^{2}\left(\mathbb{Q}_{p}\right)$ (in general, we do not assume that the functions in $\left\{f^{\left(J_{1}\right)}\right\}$ are shifts or dilations of some fixed function).

Proposition 6 Either the orbit $G f, f \in D_{0}\left(\mathbb{Q}_{p}\right)$ described in theorem 5 satisfies itself the definition (20) of the frame of wavelets, or some finite number of copies of the orbit $G f$ satisfies definition (20) of the frame of wavelets.

Proof Let us choose for $G f$ the parametrization (12): $G f=\left\{G\left(p^{\gamma} J, p^{\gamma} n\right) f\right\}$.

By the remark after the lemma 3 the orbit $G f$ with respect to the affine group of the function $f \in D_{0}\left(\mathbb{Q}_{p}\right)$ is a tight frame $\left\{G\left(p^{\gamma} J, p^{\gamma} n\right) f\right\}$ which possesses the parametrization (12). Here the scale $\gamma$ takes integer values as in (20), the translations $n \in \mathbb{Q}_{p} / p^{1-\gamma_{0}} \mathbb{Z}_{p}$ take an infinite number of possible values and the index $J$ enumerates some finite set of dilations by $p$-adic numbers with the norm one.

The translations $n$ run in $\mathbb{Q}_{p} / p^{1-\gamma_{0}} \mathbb{Z}_{p}$, i.e. depending on $n_{0}$ either $\mathbb{Q}_{p} / p^{1-\gamma_{0}} \mathbb{Z}_{p}$ is a factor group of $\mathbb{Q}_{p} / \mathbb{Z}_{p}$, or $\mathbb{Q}_{p} / \mathbb{Z}_{p}$ is a factor group of $\mathbb{Q}_{p} / p^{1-\gamma_{0}} \mathbb{Z}_{p}$, in both cases the index of the factor group (number of elements in an equivalence class) will be finite.

Consider the following cases:

1) Let $\mathbb{Q}_{p} / \mathbb{Z}_{p}$ be a factor group of $\mathbb{Q}_{p} / p^{1-\gamma_{0}} \mathbb{Z}_{p}$.

Let us prove that the frame $G f$ possesses parametrization (20) for some finite set $\left\{f^{\left(J_{1}\right)}\right\}$ of functions in $D_{0}\left(\mathbb{Q}_{p}\right)$.

Let us rewrite the parametrization (12) of the orbit $G f$ in the form

$$
G\left(p^{\gamma_{1}}, p^{\gamma_{1}} n_{1}\right) f^{\left(J_{1}\right)}(x)=G\left(p^{\gamma_{1}}, p^{\gamma_{1}} n_{1}\right) f\left(\frac{x-m}{J}\right)=G\left(p^{\gamma_{1}} J, p^{\gamma_{1}}\left(n_{1}+m\right)\right) f(x) .
$$

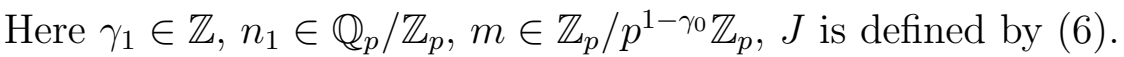

This means that $J_{1}$ takes values $(J, m)$ and

$$
f^{\left(J_{1}\right)}(x)=f\left(\frac{x-m}{J}\right) .
$$

Since the set of possible $J_{1}=(J, m)$ is finite, this proves that $G f$ satisfies definition (20).

2) Let $\mathbb{Q}_{p} / p^{1-\gamma_{0}} \mathbb{Z}_{p}$ be a factor group of $\mathbb{Q}_{p} / \mathbb{Z}_{p}$. In this case

$$
G\left(p^{\gamma_{1}}, p^{\gamma_{1}} n_{1}\right) f^{\left(J_{1}\right)}(x)=G\left(p^{\gamma_{1}}, p^{\gamma_{1}} n_{1}\right) f\left(\frac{x}{J_{1}}\right)
$$

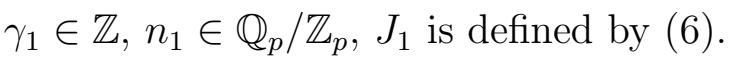


Since $f$ is invariant under the action of translations from $p^{1-\gamma_{0}} \mathbb{Z}_{p} / \mathbb{Z}_{p}$, the above set of functions coincides with the orbit $G f$ taken $p^{\gamma_{0}-1}$ times. Therefore (21) is a tight frame.

This finishes the proof of the proposition.

The above proposition is related to the fact that for any generic $f \in D_{0}\left(\mathbb{Q}_{p}\right)$ the stabilizer of the action of the affine group has a similar form and therefore the orbits of any $f \in D_{0}\left(\mathbb{Q}_{p}\right)$ possess a simple parametrization. Let us stress that this observation is related to the strong triangle inequality which is the crucial property of $p$-adic analysis.

Let us discuss the relation of the constructed frames to $p$-adic multiresolution analysis. The following $p$-adic analogue of the multiresolution approximation was discussed in [11], [8].

Definition $7 \quad A$ multiresolution approximation of $L^{2}\left(\mathbb{Q}_{p}\right)$ is a decreasing sequence $V_{\gamma}, \gamma \in \mathbb{Z}$, of closed linear subspaces of $L^{2}\left(\mathbb{Q}_{p}\right)$ with the following properties:

1)

$$
\bigcap_{-\infty}^{+\infty} V_{\gamma}=\{0\}, \quad \bigcup_{-\infty}^{+\infty} V_{\gamma} \text { is dense in } L^{2}\left(\mathbb{Q}_{p}\right) ;
$$

2) for all $f \in L^{2}\left(\mathbb{Q}_{p}\right)$ and all $\gamma \in \mathbb{Z}$,

$$
f(\cdot) \in V_{\gamma} \Longleftrightarrow f\left(p^{-1} \cdot\right) \in V_{\gamma-1} ;
$$

3) for all $f \in L^{2}\left(\mathbb{Q}_{p}\right)$ and all $n \in \mathbb{Q}_{p} / \mathbb{Z}_{p}$,

$$
f(\cdot) \in V_{0} \Longleftrightarrow f(\cdot-n) \in V_{0} ;
$$

4) there exists a function $\phi \in V_{0}$ (called the scaling function) such that the sequence

$$
\phi(\cdot-n), \quad n \in \mathbb{Q}_{p} / \mathbb{Z}_{p}
$$

is an orthonormal basis of the space $V_{0}$.

The orthogonal complement in the space $V_{\gamma-1}$ of the subspace $V_{\gamma}$ is called the wavelet space $W_{\gamma}$. The space $L^{2}\left(\mathbb{Q}_{p}\right)$ is the orthogonal sum of $W_{\gamma}, \gamma \in Z$. The spaces $W_{\gamma}$ satisfy the property analogous to the property 2 of the above definition:

$$
f(\cdot) \in W_{\gamma} \Longleftrightarrow f\left(p^{-1} \cdot\right) \in W_{\gamma-1}
$$

Example An example of the multiresolution construction is given by the scaling function $\phi(x)=\Omega\left(|x|_{p}\right)$. In this case $V_{\gamma}$ contains test functions with the diameter of local constancy $p^{\gamma}$. The wavelet space $W_{\gamma}$ possesses the basis $\left\{\psi_{\gamma n j}\right\}$ of $p$-adic wavelets with the fixed index $\gamma$ and $n \in \mathbb{Q}_{p} / \mathbb{Z}_{p}, j=1, \ldots, p-1$.

Let us compare the above definition with the structure of the orbit of generic $f \in D_{0}\left(\mathbb{Q}_{p}\right)$ with respect to action of the affine group.

In the case of the $p$-adic wavelet $f=\psi$ we get the orbit $\left\{e^{2 \pi i p^{-1} m} \psi_{\gamma n j}\right\}$. Roughly speaking this orbit is given by $p$ copies of the basis of $p$-adic wavelets. Defining $W_{\gamma}$ as the linear span of 
vectors $e^{2 \pi i p^{-1} m} \psi_{\gamma n j}$ (elements of the orbit with fixed $\gamma$ ) we reproduce the above example of $p$-adic multiresolution analysis.

In the general case $f \in D_{0}\left(\mathbb{Q}_{p}\right)$ the orbit by theorem 5 is given by the set $\left\{G\left(p^{\gamma} J, p^{\gamma} J n\right) f\right\}$.

Let us define the wavelet space $W_{-\gamma}$ as the linear span of vectors $G\left(p^{\gamma} J, p^{\gamma} J n\right) f$ with fixed $\gamma \in \mathbb{Z}$. In this case the wavelet spaces $W_{\gamma}, W_{\gamma^{\prime}}$ are not necessarily orthogonal. The spaces $W_{\gamma}$, $W_{\gamma^{\prime}}$ are orthogonal if $\left|\gamma-\gamma^{\prime}\right|$ is sufficiently large, namely, it is larger than the difference of the maximal and the minimal scales $\gamma_{1}$ of the wavelets in the expansion

$$
f=\sum_{\gamma_{1} n_{1} j_{1}} C_{\gamma_{1} n_{1} j_{1}} \psi_{\gamma_{1} n_{1} j_{1}}
$$

The property (22) for the spaces $W_{\gamma}$ will be satisfied.

We see that the frame $\left\{G\left(p^{\gamma} J, p^{\gamma} J n\right) f\right\}$ satisfies the main properties of the $p$-adic multiresolution analysis and satisfies some non-trivial generalization of the orthogonality property. Therefore it is natural to consider the structure of this frame as a generalization of the multiresolution construction.

In particular, in the case $(19)$ where $f \in D_{0}\left(\mathbb{Q}_{p}\right)$ is a linear combination of wavelets with a fixed scale, the spaces $W_{\gamma}$ will be orthogonal.

Remark The $p$-adic affine group can be considered as the inverse (projective) limit of the factor groups

$$
G=\lim _{\longleftarrow} G / G_{f}
$$

where the sequence of $f \in D_{0}\left(\mathbb{Q}_{p}\right)$ is such that the corresponding stabilizers $G_{f}$ tend to the trivial subgroup in $G$ consisting of the unity element.

This limit corresponds to the joint limit $\gamma_{A} \rightarrow+\infty, \gamma_{0} \rightarrow-\infty$, where $\gamma_{A}, \gamma_{0}$ are defined by (3), (44) correspondingly.

The multiresolution construction corresponds to the element of the sequence $\left\{G / G_{f}\right\}$ with $\gamma_{A}=1, \gamma_{0}=0$. In particular, the corresponding $f$ can be chosen according to $f(x)=\psi(x)=$ $\chi\left(p^{-1} x\right) \Omega\left(|x|_{p}\right)$.

Therefore the multiresolution construction in $p$-adic analysis can be naturally considered as a term in the sequence of the inverse limit, and we can consider other terms in this sequence as generalizations of the multiresolution analysis.

\section{Appendix}

Let us recall some constructions of $p$-adic analysis, see [12] for details and, e.g. [13], [14] for applications. The field $\mathbb{Q}_{p}$ of $p$-adic numbers is the completion of $\mathbb{Q}$ with respect to the $p$-adic norm $|\cdot|_{p}$, defined as follows. For any rational number we consider the representation

$$
x=p^{\gamma} \frac{m}{n}
$$

where $p$ is a prime, $\gamma$ is an integer, $p, m, n$ are mutually prime numbers, $n \neq 0$. The $p$-adic norm is defined as follows

$$
|x|_{p}=p^{-\gamma}
$$


A $p$-adic number can be uniquely represented by the series

$$
x=\sum_{j=\gamma}^{\infty} x_{j} p^{j}, \quad x_{j}=0, \ldots, p-1
$$

which converges in the $p$-adic norm.

A complex valued character $\chi: \mathbb{Q}_{p} \rightarrow \mathbb{C}$ of a $p$-adic argument (where $x$ has the form of the above series) is defined by

$$
\chi(x)=\exp \left(2 \pi i \sum_{j=\gamma}^{-1} x_{j} p^{j}\right), \quad \chi(x+y)=\chi(x) \chi(y) .
$$

When the above $\gamma$ is nonnegative the sum above is equal to zero and the character is equal to one.

The character $\chi$ is a locally constant function. The function $f$ is locally constant if for any $x$ there exists $\varepsilon$ for which $\forall y:|x-y|_{p} \leq \varepsilon$ we have $f(x)=f(y)$.

Another example of a locally constant function is the characteristic function of a $p$-adic ball. We denote by $\Omega(x)$ the characteristic function of the interval $[0,1]$. The characteristic function of the $p$-adic ball of radius 1 with center in 0 has the form:

$$
\Omega\left(|x|_{p}\right)= \begin{cases}1, & |x|_{p} \leq 1 \\ 0, & |x|_{p}>1\end{cases}
$$

Let us note that, unlike in the real case, the characteristic function of a $p$-adic ball is continuous (the same holds for an arbitrary locally constant function).

The $p$-adic wavelet $\psi$ is the product of character and characteristic function of the ball

$$
\psi(x)=\psi\left(p^{-1} x\right) \Omega\left(|x|_{p}\right) .
$$

The Bruhat-Schwartz space $D\left(\mathbb{Q}_{p}\right)$ of $p$-adic test functions is the linear space of locally constant complex valued functions with compact support. Any function in $D\left(\mathbb{Q}_{p}\right)$ is a (finite) linear combination of characteristic functions of balls.

The space $L^{2}\left(\mathbb{Q}_{p}\right)$ is the Hilbert space of complex valued functions which are square integrable with respect to the Haar measure on $\mathbb{Q}_{p}$. For the Haar measure on $\mathbb{Q}_{p}$ the volume of the ball is equal to the diameter of this ball.

The next theorem describes the basis of $p$-adic wavelets [5].

Theorem 8 The set of functions $\left\{\psi_{\gamma n j}\right\}$ :

$$
\begin{gathered}
\psi_{\gamma n j}(x)=p^{-\frac{\gamma}{2}} \chi\left(p^{-1} j\left(p^{\gamma} x-n\right)\right) \Omega\left(\left|p^{\gamma} x-n\right|_{p}\right), \\
\gamma \in \mathbb{Z}, \quad j=1, \ldots, p-1, \quad n \in \mathbb{Q}_{p} / \mathbb{Z}_{p}, \\
n=\sum_{l=-\delta}^{-1} n_{l} p^{l}, \quad n_{l}=0, \ldots, p-1,
\end{gathered}
$$

is an orthonormal basis in $L^{2}\left(\mathbb{Q}_{p}\right)$ (the basis of p-adic wavelets). 
In the present paper the notation $n \in \mathbb{Q}_{p} / \mathbb{Z}_{p}$ always means that we choose for $n$ the representative in the corresponding equivalence class of the form (24). More generally, for $n \in \mathbb{Q}_{p} / p^{\gamma_{0}} \mathbb{Z}_{p}$ we always choose the representative

$$
n=\sum_{l=-\delta}^{\gamma_{0}-1} n_{l} p^{l}, \quad n_{l}=0, \ldots, p-1 .
$$

The space $D_{0}\left(\mathbb{Q}_{p}\right)$ is the space of mean zero test functions. Any function in $D_{0}\left(\mathbb{Q}_{p}\right)$ is a (finite) linear combination of $p$-adic wavelets:

$$
f=\sum_{\gamma n j} C_{\gamma n j} \psi_{\gamma n j}, \quad C_{\gamma n j} \in \mathbb{C} .
$$

Definition 9 The set of vectors $\left\{f_{n}\right\}$ in the Hilbert space $\mathcal{H}$ is a frame, if there exist positive constants $A, B>0$, such that for each vector $g \in \mathcal{H}$ the following inequality is satisfied:

$$
A\|g\|^{2} \leq \sum_{n}\left|\left\langle g, f_{n}\right\rangle\right|^{2} \leq B\|g\|^{2} .
$$

The constants $A$ and $B$ are called the lower and the upper bounds of the frame correspondingly. A frame is tight if the frame bounds $A$ and $B$ are equal. A frame is uniform if all elements have equal norms.

The unitary representation in $L^{2}\left(\mathbb{Q}_{p}\right)$ of the $p$-adic affine group $G$ acts on the function $f \in$ $L^{2}\left(\mathbb{Q}_{p}\right)$ by translations and dilations

$$
G(a, b) f(x)=f^{a, b}(x)=|a|_{p}^{-\frac{1}{2}} f\left(\frac{x-b}{a}\right),
$$

where $a, b \in \mathbb{Q}_{p}$ and $a \neq 0$.

The composition of the elements of the affine group has the form

$$
G(a, b) G\left(a^{\prime}, b^{\prime}\right)=G\left(a a^{\prime}, b+a b^{\prime}\right) .
$$

This implies for the degree of $G(a, b)$

$$
G^{n}(a, b)=G\left(a^{n}, b[n]_{a}\right) .
$$

Here $[n]_{1}=n$ and for $a \neq 1$

$$
[n]_{a}=\frac{1-a^{n}}{1-a} .
$$

The inverse to $G(a, b)$ has the form:

$$
G^{-1}(a, b)=G\left(a^{-1},-b a^{-1}\right) .
$$

Acknowledgments One of the authors (S.K.) would like to thank I.V.Volovich, V.S.Vladimirov and A.Yu.Khrennikov for fruitful discussions and valuable comments. He gratefully acknowledges being partially supported by the grant DFG Project 436 RUS 113/809/0-1 and DFG Project 436 RUS 113/951, by the grants of the Russian Foundation for Basic Research RFFI 0501-04002-NNIO-a and RFFI 08-01-00727-a, by the grant of the President of Russian Federation for the support of scientific schools NSh 3224.2008.1 and by the Program of the Department of Mathematics of the Russian Academy of Science "Modern problems of theoretical mathematics". He is also grateful to IZKS (the Interdisciplinary Center for Complex Systems) of the University of Bonn for kind hospitality. 


\section{References}

[1] I.Daubechies Ten Lectures on Wavelets, CBMS Lecture Notes Series. SIAM, Philadelphia, 1992.

[2] Y.Meyer Wavelets and operators, Cambridge University Press, Cambridge, 1992.

[3] S.G. Mallat A Wavelet Tour of Signal Processing, Academic Press, 1999

[4] I. Ya. Novikov, V.Yu. Protasov, M.A. Skopina Wavelet Theory. Moscow: Fizmatlit, 2005. (in Russian)

[5] S.V.Kozyrev Wavelet theory as p-adic spectral analysis // Izvestiya: Mathematics. 2002. V.66. N.2. P.367-376. http://arxiv.org/abs/math-ph/0012019

[6] J.J. Benedetto, R.L. Benedetto A wavelet theory for local fields and related groups // The Journal of Geometric Analysis. 2004. V.14. N.3. P.423-456.

[7] R.L.Benedetto Examples of wavelets for local fields // Contemporary Mathematics. 2004. V.345. AMS, Providence. P.27-47. http://arxiv.org/math.CA/abs/0312038

[8] V.M.Shelkovich, M.Skopina p-Adic Haar multiresolution analysis, http://arxiv.org/abs/0704.0736

[9] A.Yu. Khrennikov, V.M. Shelkovich, M. Skopina p-Adic refinable functions and MRA-based wavelets, http://arxiv.org/abs/0711.2820

[10] S.Albeverio, A.Yu.Khrennikov, V.M.Shelkovich Harmonic analysis in the $p$-adic Lizorkin spaces: fractional operators, pseudo-differential equations, $p$-adic wavelets, Tauberian theorems // The Journal of Fourier Analysis and Applications. 2006. V.12. N.4. P.393-425.

[11] S.Albeverio, S.V.Kozyrev Coincidence of the continuous and discrete $p$-adic wavelet transforms, http://arxiv.org/abs/math-ph/0702010

[12] V.S. Vladimirov, I.V.Volovich, Ye.I.Zelenov p-Adic Analysis and Mathematical Physics. Singapore: World Scientific, 1994.

[13] S.Albeverio, W.Karwowski A random walk on $p$-adics - the generator and its spectrum // Stoch. Processes Appl. 1994. V.53. no.1. P.1-22.

[14] A.Yu.Khrennikov, M.Nilsson P-adic Deterministic and Random Dynamics, Kluwer Academic, Dordrecht-Boston-London, 2004. 